\title{
Dual-Polarized Dual-Band Antenna Element for ISM Bands
}

\author{
W.M. Dorsey ${ }^{1,2^{\star}}$, A.I. Zaghloul ${ }^{1,3}$ \\ ${ }^{1}$ Virginia Polytechnic Institute and State University: ECE Dept. \\ Falls Church, VA \\ wmdorsey@vt.edu, amirz@vt.edu \\ ${ }^{2}$ US Naval Research Laboratory: Radar Division \\ Washington, DC \\ dorsey@radar.nrl.navy.mil \\ ${ }^{3}$ US Army Research Lab \\ Adelphi, MD \\ Amir.zaghloul@arl.army.mil
}

\begin{abstract}
A dual-band element that operates in two separate industrial, scientific, and medical (ISM) bands is presented that provides dual-linear or dual-circular polarization in each band. The element is ideal for wireless communication applications utilizing multiple protocol and/or Bluetooth technology. This design is realizable in cost effective printed circuit board technology making it an attractive design for low cost personal communications devices.
\end{abstract}

\section{INTRODUCTION}

Wireless access points and laptops are both turning towards antennas capable of operating in multiple frequency bands in order to support multiple protocols [1-3]. The $2.4 \mathrm{GHz}$ industrial, scientific, and medical (ISM) band is quickly growing in popularity for wireless communications devices due to its use in Bluetooth technology and $802.11 \mathrm{~b} / \mathrm{g}$ protocol. For higher data rates, the frequency band from $5.15-5.85 \mathrm{GHz}$ is often used, and the 802.11a protocol operates within the 5.2GHz ISM band [2].

Circular polarized (CP) antennas are popular choices in wireless communications applications owing to their ability to allow flexible orientation in the plane of the transmitter and receiver antennas and to reduce multipath effects that can lead to signal fading [4-6]. The ability to operate with both senses of CP allows frequency reuse to double system capacity [7]. In two-way data link systems, information is often transmitted by means of polarization shift keying, a technique that utilizes orthogonal senses of CP [7]. This paper presents a dual-ISM band antenna element that allows each band to operate with either dual-linear or dual-circular polarization.

\section{DUAL-BAND DUAL-POLARIZED ELEMENT TOPOLOGY}

A dual-band dual-CP element is illustrated in Figure 1. The low band shorted square ring has an outer side length of $\mathrm{L}_{0}$ and an inner side length of $\mathrm{L}_{1}$. The high band square ring slot has outer and inner side lengths of $L_{1}$ and $L_{2}$ respectively. The top view of Figure 1(b) also shows the isosceles triangle shaped perturbations at opposing corners of both radiators to generate single-feed CP. By having two orthogonal feed points for each 
band, this dual-band element is capable of generating dual-CP operation for each frequency band. For applications requiring dual-linear polarization, no perturbations would be present on the conductors.

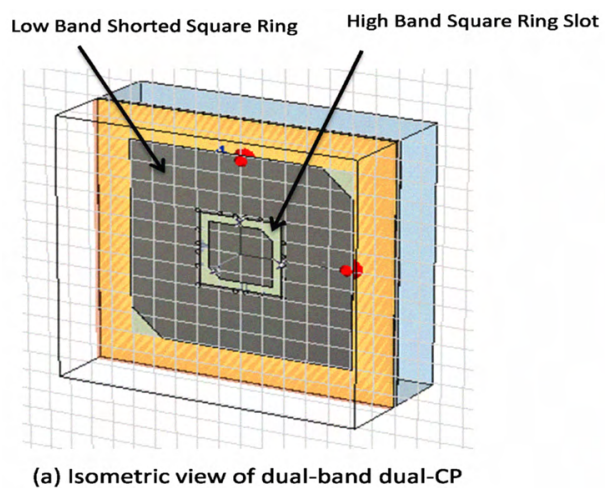

(a) Isometric view of dual-band dual-CP element

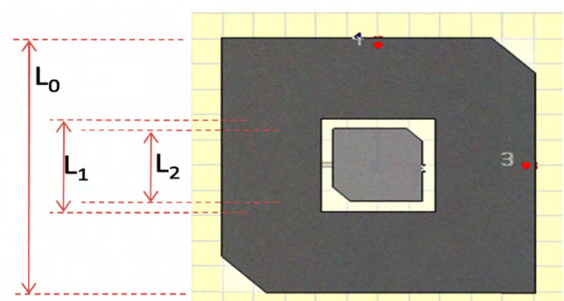

(b) Top view of dual-band dual-CP element

Figure 1: Illustration of Dual-Band Dual-CP Antenna Element

The high band slot element is fed with orthogonal feed lines that are terminated in open circuit stubs. The two feed lines are printed on opposing sides of a thin substrate layer to avoid physical intersection. The feed substrate is then sandwiched between two other substrate layers and conductors are present on the top and bottom of the sandwiched dielectric profile.

The stripline feeds for exciting the high band element must pass through the plated through holes that provide the shorting mechanism for the low band element. In addition to shorting the low band element, the plated through holes act as mode suppressors for the parallel plate mode that can cause lower efficiency in slotted stripline designs $[8,9]$. The stripline feeds for the high band are transitioned to a microstrip layer present beneath the antenna ground plane. Figures 2(a)-(c) illustrate this transition occurring just outside of the square ring slot. A plated through hole provides electrical continuity between the stripline and a microstrip transmission line of matched characteristic impedance.
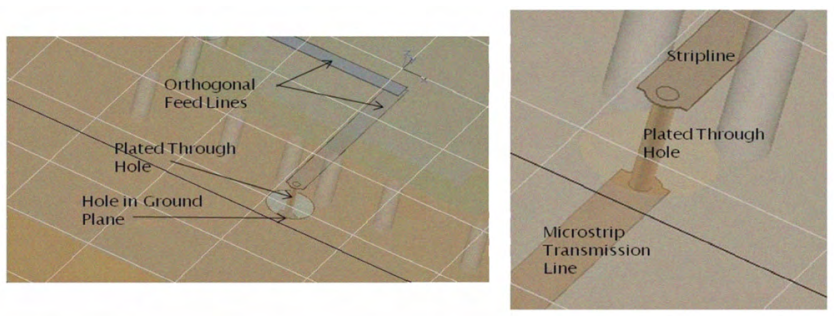

(a) Isometric view of orthogonal feed lines (b) Isometric view of stripline-tomicrostrip transition

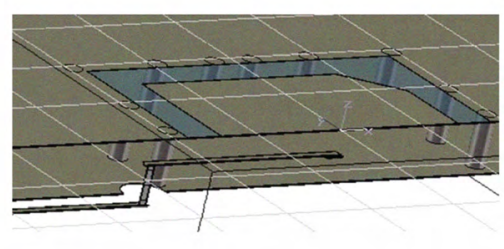

(c) Isometric section view of stripline-to-microstrip transition

Figure 2: Section view of Stripline-to-microstrip transitions

The low band element is fed by orthogonal feed probe. These feed probes can be realized as coaxial probe feeds or plated through holes from transmission lines present on the microstrip layer that contains the feeding microstrip lines for the high band element. 


\section{SIMULATION RESULTS}

An element was designed with the goal to cover the $2.45 \mathrm{GHz}$ and $5.8 \mathrm{GHz}$ ISM bands with dual-CP operation at each band. The simulations for this element were carried out using CST Microwave Studio [10], a computational electromagnetic tool using the Finite Integration Technique (FIT). This element can be viewed as a four-port network; Ports 1 and 2 are the high band RHCP (Right Hand Circularly Polarized) and LHCP (Left Hand Circularly Polarized) port respectively, and Ports 3 and 4 are the low band LHCP and RHCP ports. A detailed look into the s-parameters of the four-port antenna is provided in Figure 3(a)-(d). The s-parameters show that the isolation is greater than $25 \mathrm{~dB}$ between the high and low band ports. The isolation between the two high band ports $\left(\left|\mathbf{s}_{21}\right|,\left|\mathbf{s}_{12}\right|\right)$ has a maximum value greater than $40 \mathrm{~dB}$ at the center of the band. The port-to-port isolation between the low band ports $\left(\left|s_{43}\right|,\left|s_{34}\right|\right)$ is lower than that of the high band ports. This finding is similar to that in the literature for dual-polarized microstrip patch antennas. When the corners of the patch are perturbed to achieve dual-CP operation, the orthogonal modes couple strongly to each other [11]. It has also been shown that this port-to-port isolation can be increased at the expense of impedance match and axial ratio $[7,11]$.

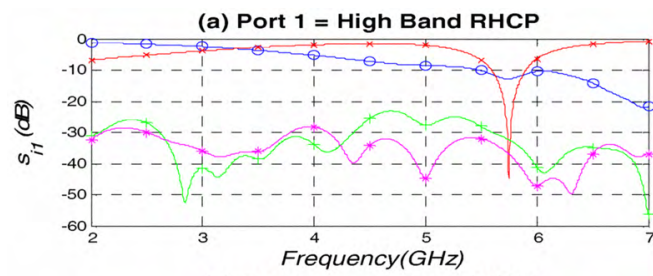

(c) Port 3 = Low Band LHCP

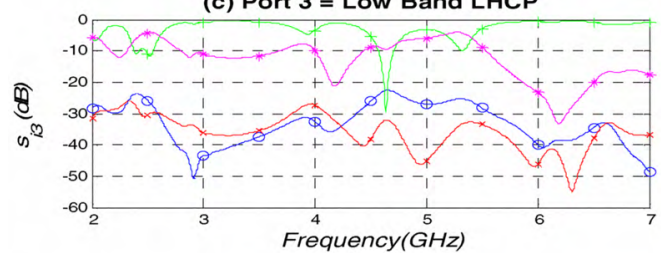

Figure 3: Simulated s-parameters for Dual-Band Dual-CP Antenna Element (b) Port 2 = High Band LHCP

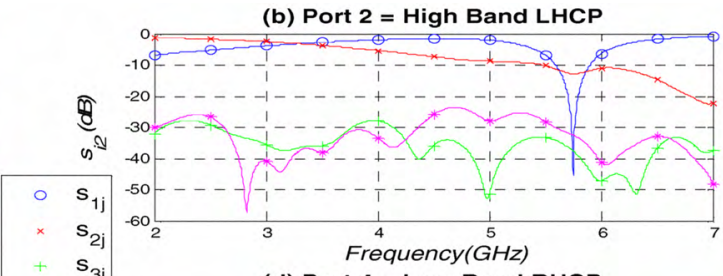

(d) Port 4 = Low Band RHCP

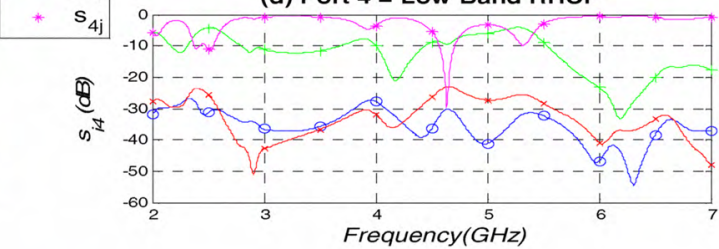

The axial ratio for the low and high band ports is plotted in Figure 4 as a function of frequency and theta. The low band has a minimum axial ratio of $0.33 \mathrm{~dB}$ occurring at $2.44 \mathrm{GHz}$, and the axial ratio is below $3 \mathrm{~dB}$ over the majority of the $2.45 \mathrm{GHz}$ ISM band. The high band element has a broader CP bandwidth (8.5\%), which is typical of slot elements. The high band element has a minimum axial ratio of $0.32 \mathrm{~dB}$ for RHCP and $0.89 \mathrm{~dB}$ for LHCP occurring at $5.9 \mathrm{GHz}$. The high band element has an axial ratio better than $3 \mathrm{~dB}$ from $5.6-6.1 \mathrm{GHz}$, a bandwidth of $8.5 \%$. The axial ratio holds up over a wide range of theta values which is critical in applications requiring high performance at angles other than broadside. 


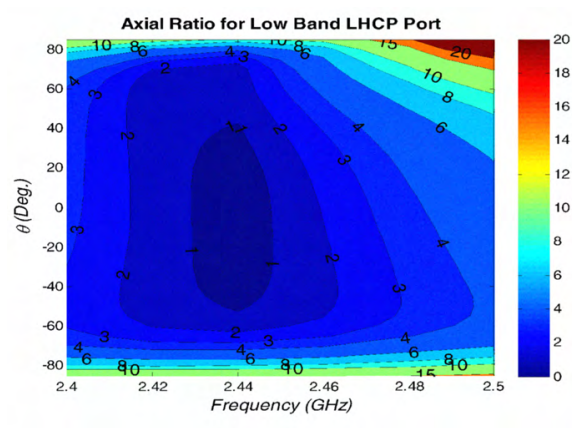

(a) Low Band

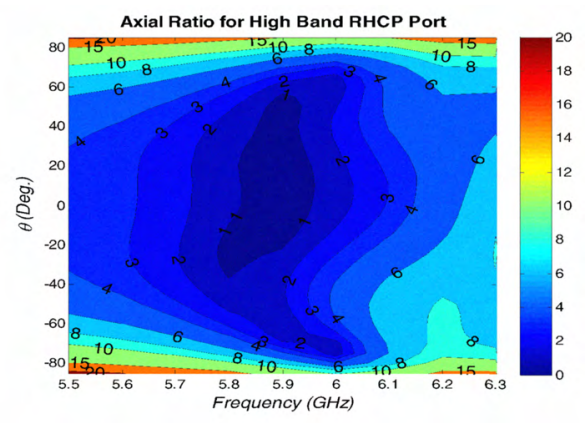

(b) High Band

Figure 4: Dual-Band, Dual-CP Axial Ratio

\section{CONCLUSION}

The element proposed has the capability to operate with orthogonal polarizations in two frequency bands. A printed circuit design provides a low profile, light weight, and low cost design that makes is desirable for integration with laptop technology, wireless access points, space born radars, cellular phone handsets and bases stations, and many other areas of the ever growing field of wireless communications.

\section{REFERENCES}

[1] Lindmark, B., "A dual polarized dual band microstrip antenna for wireless communications," Aero. Conf, 1998. Proc., IEEE , vol.3, no., pp.333-338 vol.3, 21-28 Mar 1998.

[2] Duxian Liu; Gaucher, B., "A new multiband antenna for WLAN/cellular applications," Vehicular Technology Conference, 2004. VTC2004-Fall. 2004 IEEE 60th, vol.1, no., pp. 243-246 Vol. 1, 26-29 Sept. 2004.

[3] Bialkowski, K.S.; Zagriatski, S., "Investigations into a dual band $2.4 / 5.2 \mathrm{GHz}$ antenna for WLAN applications," Microwaves, Radar and Wireless Communications, 2004. MIKON-2004. 15th Int. Conf on, vol.2, no., pp. 660-663 Vol.2, 17-19 May 2004.

[5] Hsu, S.H.; Chang, K., "A Novel Reconfigurable Microstrip Antenna with Switchable Circular Polarization", IEEE Ant. and Wireless Prop. Let., Vol. 6, 2007, pp. 160-162.

[6] Sung, Y.J.; Jang, T.U.; Kim, Y.S., "A Reconfigurable Microstrip Antenna for Switchable Polarization", IEEE Micro. \& Wireless Comp. Let., Vol. 14, Nov. 2004, pp. 534-536.

[7] Fang, S.T., "A Novel Polarization Diversity Antenna for WLAN Applications", Antennas and Prop. Society International Symp., Vol. 1, 16-21 July 2000, pp. 282-285.

[8] Jefferson, R.L.; Smith, D., "Dual circular polarised microstrip antenna design for a passive microwave transponder," Antennas and Propagation, 1991. ICAP 91., Seventh International Conference on (IEE) , vol., no., pp.141-143 vol.1, 15-18 Apr 1991.

[9] Itoh, K; Yamamoto, M., "A Slot Coupled Microstrip Array Antenna with a Triplate Line Feed where Parallel-Plate Mode is Suppressed Efficiently", Antennas and Prop. Society International Symp., Vol. 4, 13-18 July 1997, pp. 2135-2138.

[10] Bhattacharyya, A; Fordham, O; Liu, Y., "Analysis of Stripline-Fed Slot-Coupled Patch Antennas with Vias for Parallel-Plate Mode Suppression", IEEE Transactions on Antennas and Propagation, Vol. 46, No. 4, April 1998, pp. 538-545.

[11] CST Microwave Studio.

[12] Owens, R.P.; Smith, A.C., "Low-profile dual band, dual polarised array antenna module," Electronics Letters, vol.26, no.18, pp.1433-1434, 30 Aug. 1990. 